\title{
451 - Estimating "Brain Age Gaps" in patients with brain injury: Applying machine learning to advanced neuroimaging techniques
}

Introduction: A single moderate or severe TBI is associated with accelerated brain aging and increased risk for dementia. Despite the high rate of falls that result in brain injury in older adults, numerous factors such as genetic predisposition to Alzheimer's disease, sex, education, age are also known to affect multiple age-sensitive neuroimaging markers.

METHODS: Here we use the "brain age" metric being tested by the global consortium, Enhancing Neurolmaging Genetics through Meta-Analysis (ENIGMA), that employs machine learning to predict a person's age from multiple age-sensitive imaging markers (e.g., hippocampal volume, regional cortical gray matter thickness, intracranial volume (ICV)), while also taking into account their sex and educational level. We will discuss results from brain injured patients ( $n=60$; age range: $20-75$ years) and healthy age-matched controls ( $n=20$ (20-75 years). We will compute the "brain age gap" - between a person's actual chronological age and that predicted from their brain scan - and test relations between this measure and injury characteristics.

RESULTS: In our pilot work, we predicted a person's age from their MRI scan with a mean absolute error of about 5 years. ENIGMA's current best model includes: (1) non-normalized brain volumetric measures as predictors including ICV, (2) separate models for males and females, (3) use of a large age range (1280), and (4) Gaussian process regression (GPR).

CONCLUSION: This "overall" marker of accelerated brain aging offers a metric that taps diverse sources of information, weighted by their relevance to brain aging, and is associated with decreased functionality in older adults. 